\title{
MEAN SQUARE CONTINUITY OF ORNSTEIN-UHLENBECK PROCESSES IN BANACH SPACES
}

\author{
Z. BRZEŹNIAK, B. GOLDYS, AND J.M.A.M. VAN NEERVEN
}

Abstract. Let $X\left(t, x_{0}\right)$ denote the weak solution of the stochastic abstract Cauchy problem

$$
\begin{aligned}
d X(t) & =A X(t) d t+B d W_{H}(t), \quad t \geq 0, \\
X(0) & =x_{0} .
\end{aligned}
$$

Here $A$ generates a $C_{0}$-semigroup on a separable real Banach space $E$, $\left\{W_{H}(t)\right\}_{t \geq 0}$ is a cylindrical Wiener process with Cameron-Martin space $H, B \in \mathcal{L}(H, E)$ is a bounded linear operator, and $x_{0} \in E$ is a given intitial value. We prove that for all $p \in[1, \infty)$ and $t \geq 0$,

$$
\lim _{s \rightarrow t} \mathbb{E}\left(\left\|X\left(t, x_{0}\right)-X\left(s, x_{0}\right)\right\|^{p}\right)=0 \text {. }
$$

We consider the following stochastic abstract Cauchy problem:

$$
\begin{aligned}
d X(t) & =A X(t) d t+B d W_{H}(t), \quad t \geq 0, \\
X(0) & =x_{0},
\end{aligned}
$$

where $A$ is the generator of a $C_{0}$-semigroup $\mathbf{S}=\{S(t)\}_{t \geq 0}$ on a separable real Banach space $E, B$ is a bounded linear operator from a separable real Hilbert space $H$ into $E$, and $\left\{W_{H}(t)\right\}_{t \geq 0}$ is a cylindrical Wiener process with Cameron-Martin space $H$. For the precise definition of this concept we refer to [3].

It has been shown in [3] that the problem (1.1) admits a weak solution $\left\{X\left(t, x_{0}\right)\right\}_{t \geq 0}$ if and only if for each $t>0$ the operator $Q_{t} \in \mathcal{L}\left(E^{*}, E\right)$ defined by

$$
Q_{t} x^{*}:=\int_{0}^{t} S(s) B B^{*} S^{*}(s) x^{*} d s, \quad x^{*} \in E^{*},
$$

is the covariance of a centred Gaussian measure $\mu_{t}$ on $E$. In this case, $\mu_{t}$ is the distribution of the random variable $X(t, 0)$, and the solution can be represented as a stochastic convolution as follows:

$$
\left\langle X\left(t, x_{0}\right), x^{*}\right\rangle=\left\langle S(t) x_{0}, x^{*}\right\rangle+\int_{0}^{t}\left\langle S(t-s) B d W_{H}(s), x^{*}\right\rangle, \quad x^{*} \in E^{*} .
$$

2000 Mathematics Subject Classification. 35R15, 60H15, 47D06.

Key words and phrases. mean square continuous, Ornstein-Uhlenbeck process, stochastic Cauchy problem. 
We will prove that the process $\left\{X\left(t, x_{0}\right)\right\}_{t \geq 0}$ is mean continuous in all moments. In particular this solves the problem, left open in [3], whether $\left\{X\left(t, x_{0}\right)\right\}_{t \geq 0}$ is mean square continuous.

Let $C_{b}(E)$ denote the space of all bounded continuous real functions on $E$.

Lemma 1. Let $\left(t_{n}\right)$ be a sequence of nonnegative real numbers in the interval $[0, T]$ with $\lim _{n \rightarrow \infty} t_{n}=t$. Let $g:[0, \infty) \rightarrow \mathbb{R}$ be nondecreasing and convex with $g(\|\cdot\|) \in L^{1}\left(E, \mu_{T}\right)$. Then for all $f \in C_{b}(E)$ we have

$$
\lim _{n \rightarrow \infty} \int_{E} f(x) g(\|x\|) d \mu_{t_{n}}(x)=\int_{E} f(x) g(\|x\|) d \mu_{t}(x) .
$$

Proof. For $r>0$ let

$$
B_{r}=\{x \in E: g(\|x\|) \leq r\}
$$

This set is symmetric and convex. Symmetry is obvious, and convexity follows from

$g(\|\alpha x+(1-\alpha) y\|) \leq g(\alpha\|x\|+(1-\alpha)\|y\|) \leq \alpha g(\|x\|)+(1-\alpha) g(\|y\|)$,

where $\alpha \in[0,1]$. In view of

$$
\left\langle Q_{t_{n}} x^{*}, x^{*}\right\rangle \leq\left\langle Q_{T} x^{*}, x^{*}\right\rangle, \quad x^{*} \in E^{*},
$$

we may apply Anderson's inequality [2, Theorem 3.3.6] to obtain

$$
\mu_{t_{n}}\left(B_{r}\right) \geq \mu_{T}\left(B_{r}\right)
$$

In combination with the identity

$$
\int_{E}|h(x)| d \nu(x)=\int_{0}^{\infty} \nu\{x \in E:|h(x)|>s\} d s,
$$

we find, with $M=\sup _{x \in E}|f(x)|$,

$$
\begin{aligned}
\int_{E \backslash B_{r}}|f(x)| g(\|x\|) d \mu_{t_{n}}(x) & \leq M \int_{E \backslash B_{r}} g(\|x\|) d \mu_{t_{n}}(x) \\
& =M \int_{g(r)}^{\infty} \mu_{t_{n}}\{x \in E: g(\|x\|)>s\} d s \\
& \leq M \int_{g(r)}^{\infty} \mu_{T}\{x \in E: g(\|x\|)>s\} d s \\
& =M \int_{E \backslash B_{r}} g(\|x\|) d \mu_{T}(x) .
\end{aligned}
$$


The same argument gives

$$
\int_{E \backslash B_{r}}|f(x)| g(\|x\|) d \mu_{t}(x) \leq M \int_{E \backslash B_{r}} g(\|x\|) d \mu_{T}(x) .
$$

It now follows easily that the family $f\left(X\left(t_{n}, 0\right)\right) g\left(\left\|X\left(t_{n}, 0\right)\right\|\right)$ is uniformly integrable. Since $\mu_{t_{n}} \rightarrow \mu_{t}$ weakly [6], the lemma follows from [1, Theorem 5.4]. Alternatively, the weak convergence $\mu_{t_{n}} \rightarrow \mu_{t}$ implies

$$
\lim _{n \rightarrow \infty} \int_{E} f(x)(g(\|x\|) \wedge g(r)) d \mu_{t_{n}}(x)=\int_{E} f(x)(g(\|x\|) \wedge g(r)) d \mu_{t}(x)
$$

for all $r>0$. Choosing $r$ so large that $\int_{E \backslash B_{r}} g(\|x\|) d \mu_{T}(x)<\varepsilon / M$, by (1.2) and (1.3) both truncation errors are at most $\varepsilon$, and again the lemma follows.

If $f: E \rightarrow \mathbb{R}$ is a bounded Borel function, then for all $t \geq 0$ we have

$$
\mathbb{E}(f(X(t, 0)))=\int_{E} f(y) d \mu_{t}(y) .
$$

By an easy approximation argument, for $t \geq 0$ fixed this identity extends to all functions $f \in L^{1}\left(E, \mu_{t}\right)$.

Theorem 2. Let $g:[0, \infty) \rightarrow \mathbb{R}$ be a nondecreasing convex function with $g(0)=0$ such that

$$
g(c\|\cdot\|) \in L^{1}\left(E, \mu_{T}\right)
$$

for some $c>M+2$, where $M=\lim \sup _{u \downarrow 0}\|S(u)\|$. Then for all $x \in E$ and $t \in[0, T]$ we have

$$
\lim _{s \rightarrow t} \mathbb{E} g(\|X(t, x)-X(s, x)\|)=0 .
$$

Proof. The assumption $c>M+2$ enables us to choose $\alpha, \beta, \gamma \in(0,1)$ with $\alpha+\beta+\gamma=1$ subject to the following two conditions:

- $\beta c>M+1$;

- $\gamma c \geq 1$.

Step 1 - First we note that for all $\tau \in[0, T]$ and $0 \leq c^{\prime} \leq c$,

$$
\begin{aligned}
\int_{E} g\left(c^{\prime}\|y\|\right) d \mu_{\tau}(y) & =\int_{0}^{\infty} \mu_{\tau}\left\{x \in E: g\left(c^{\prime}\|x\|\right)>s\right\} d s \\
& \leq \int_{0}^{\infty} \mu_{T}\left\{x \in E: g\left(c^{\prime}\|x\|\right)>s\right\} d s \\
& =\int_{E} g\left(c^{\prime}\|y\|\right) d \mu_{T}(y) \\
& \leq \int_{E} g(c\|y\|) d \mu_{T}(y)<\infty
\end{aligned}
$$


Hence by the condition $1 / \gamma \leq c$ and the remark preceding the theorem,

$$
\mathbb{E} g\left(\frac{1}{\gamma}\|X(\tau, 0)\|\right)=\int_{E} g\left(\frac{1}{\gamma}\|y\|\right) d \mu_{\tau}(y),
$$

and therefore by Lemma 1,

$$
\lim _{\tau \downarrow 0} \mathbb{E} g\left(\frac{1}{\gamma}\|X(\tau, 0)\|\right)=\lim _{\tau \downarrow 0} \int_{E} g\left(\frac{1}{\gamma}\|y\|\right) d \mu_{\tau}(y)=0 .
$$

Step 2 - Right continuity. Fix $t \in[0, T]$. We have, for $t \leq s \leq T$,

$$
\begin{aligned}
&\left\langle X(s, x), x^{*}\right\rangle-\left\langle X(t, x), x^{*}\right\rangle \\
&=\left\langle S(s) x, x^{*}\right\rangle+\int_{0}^{s}\left\langle S(s-u) B d W_{H}(u), x^{*}\right\rangle \\
& \quad-\left\langle S(t) x, x^{*}\right\rangle-\int_{0}^{t}\left\langle S(t-u) B d W_{H}(u), x^{*}\right\rangle \\
&=\left\langle S(s) x-S(t) x, x^{*}\right\rangle+\left\langle S(s-t) X(t, 0)-X(t, 0), x^{*}\right\rangle \\
& \quad+\left\langle Y_{s, t}, x^{*}\right\rangle
\end{aligned}
$$

where

$$
Y_{s, t}=\int_{t}^{s} S(s-u) B d W_{H}(u)
$$

Hence,

(1.4) $X(s, x)-X(t, x)=S(s) x-S(t) x+S(s-t) X(t, 0)-X(t, 0)+Y_{s, t}$.

The convexity of $g$ implies

$$
\begin{aligned}
& g(\|X(s, x)-X(t, x)\|) \\
& \quad \leq \alpha g\left(\frac{1}{\alpha}\|S(s) x-S(t) x\|\right)+\beta g\left(\frac{1}{\beta}\|S(s-t) X(t, 0)-X(t, 0)\|\right) \\
& \quad+\gamma g\left(\frac{1}{\gamma}\left\|Y_{s, t}\right\|\right) .
\end{aligned}
$$

Noting that $g$ is continuous with $g(0)=0$, it follows that

$$
\lim _{s \downarrow t} \mathbb{E} g\left(\frac{1}{\alpha}\|S(s) x-S(t) x\|\right)=\lim _{s \downarrow t} g\left(\frac{1}{\alpha}\|S(s) x-S(t) x\|\right)=0 .
$$

Arguing as in Step 1 and using the condition $(M+1) / \beta<c$ we see that for $s-t$ sufficiently small,

$$
\mathbb{E} g\left(\frac{1}{\beta}\|S(s-t) X(t, 0)-X(t, 0)\|\right)=\int_{E} g\left(\frac{1}{\beta}\|S(s-t) y-y\|\right) d \mu_{t}(y) \text {. }
$$

Hence by dominated convergence,

$$
\begin{array}{rl}
\lim _{s \downarrow t} \mathbb{E} & g\left(\frac{1}{\beta}\|S(s-t) X(t, 0)-X(t, 0)\|\right) \\
\quad= & \lim _{s \downarrow t} \int_{E} g\left(\frac{1}{\beta}\|S(s-t) y-y\|\right) d \mu_{t}(y)=0 .
\end{array}
$$


Finally, noting that $Y_{s, t}$ and $X(s-t, 0)$ have the same distribution, by Step 1 we have

$$
\lim _{s \downarrow t} \mathbb{E} g\left(\frac{1}{\gamma}\left\|Y_{s, t}\right\|\right)=\lim _{s \downarrow t} \mathbb{E} g\left(\frac{1}{\gamma}\|X(s-t, 0)\|\right)=0 .
$$

Step 3 - Left continuity. Fix $t \in[0, T]$. For $0 \leq s \leq t$ we have, using (1.4) with the rôles of $s$ and $t$ reversed,

$$
\begin{aligned}
& g(\|X(t, x)-X(s, x)\|) \\
& \quad \leq \alpha g\left(\frac{1}{\alpha}\|S(t) x-S(s) x\|\right)+\beta g\left(\frac{1}{\beta}\|S(t-s) X(s, 0)-X(s, 0)\|\right) \\
& \quad+\gamma g\left(\frac{1}{\gamma}\left\|Y_{t, s}\right\|\right) .
\end{aligned}
$$

As in Step 2, the expectation of the first term on the right hand side tends to 0 as $s \uparrow t$ by continuity, and the expectation of the third term tends to 0 by Step 1. It remains to prove that

$$
\begin{array}{rl}
\lim _{s \uparrow t} & \mathbb{E} g\left(\frac{1}{\beta}\|S(t-s) X(s, 0)-X(s, 0)\|\right) \\
& =\lim _{s \uparrow t} \int_{E} g\left(\frac{1}{\beta}\|S(t-s) y-y\|\right) d \mu_{s}(y)=0 .
\end{array}
$$

By Lemma 1 , for all $s \in[0, T]$ the measure $g(c\|x\|) d \mu_{s}(x)$ is a finite Radon measure and the family

$$
\left\{g(c\|x\|) d \mu_{s}(x): s \in\left[\frac{1}{2} t, t\right]\right\}
$$

is tight. Fix $\varepsilon>0$ arbitrary and use Prokhorov's theorem to choose a compact set $K$ such that

$$
\int_{E \backslash K} g(c\|x\|) d \mu_{s}(x)<\varepsilon, \quad s \in\left[\frac{1}{2} t, t\right] .
$$

Choose $0<\tau \leq \frac{1}{2} t$ so small that

$$
\frac{1}{\beta}(\|S(u)\|+1) \leq c \quad \text { and } \quad \frac{1}{\beta}\|S(u) y-y\|<\varepsilon, \quad u \in[0, \tau], y \in K .
$$

It follows that for $s \in[t-\tau, t]$,

$$
\begin{aligned}
& \int_{E} g\left(\frac{1}{\beta}\|S(t-s) y-y\|\right) d \mu_{s}(y) \\
& \leq \int_{K} g\left(\frac{1}{\beta}\|S(t-s) y-y\|\right) d \mu_{s}(y) \\
& \quad+\int_{E \backslash K} g\left(\frac{1}{\beta}\|S(t-s) y-y\|\right) d \mu_{s}(y) . \\
& \leq g(\varepsilon)+\varepsilon .
\end{aligned}
$$

Since $\lim _{\varepsilon \downarrow 0} g(\varepsilon)=0$, this completes the proof. 
Under a slightly stronger assumption on $g$, we can rephrase this result in terms of Orlicz norms.

If $g:[0, \infty) \rightarrow \mathbb{R}$ is a nondecreasing convex function with $g(0)=0$, then for a strongly measurable function $\xi:(\Omega, \mathbb{P}) \rightarrow E$ we define

$$
\|\xi\|_{L_{g}(E)}:=\inf \left\{c>0: \mathbb{E} g\left(\frac{\|\xi\|}{c}\right) \leq 1\right\} .
$$

The set $L_{g}(E)$ of all $\xi$ for which $\|\xi\|_{L_{g}(E)}$ is finite is a Banach space; cf. [5].

Corollary 3. Let $g:[0, \infty) \rightarrow \mathbb{R}$ be a nondecreasing convex function with $g(0)=0$ such that $g(c\|\cdot\|) \in L^{1}\left(E, \mu_{T}\right)$ for all $c>0$. Then for all $x \in E$ and $t \in[0, T]$ we have

$$
\lim _{s \rightarrow t} X(s, x)=X(t, x) \text { in } L_{g}(E) .
$$

Proof. Let $\varepsilon>0$ be fixed and define $g_{\varepsilon}(\tau):=g\left(\varepsilon^{-1} \tau\right)$. According to Theorem 2, for $|t-s|$ sufficiently small we have

$$
\mathbb{E} g_{\varepsilon}(\|X(t, x)-X(s, x)\|) \leq 1
$$

Hence,

$$
\mathbb{E} g\left(\frac{\|X(t, x)-X(s, x)\|}{\varepsilon}\right)=\mathbb{E} g_{\varepsilon}(\|X(t, x)-X(s, x)\|) \leq 1,
$$

which means that $\|X(t, x)-X(s, x)\|_{L_{g}(E)} \leq \varepsilon$.

By Fernique's theorem, this result applies, e.g., to the functions

$$
g(\tau)=\exp \left(\tau^{p}\right)-1, \quad 1 \leq p<2,
$$

and $g(\tau)=\tau^{p}, 1 \leq p<\infty$. In the latter case we can apply Theorem 2 directly and obtain:

Corollary 4. For all $x \in E$ and $t \geq 0$ we have

$$
\lim _{s \rightarrow t} \mathbb{E}\left(\|X(t, x)-X(s, x)\|^{p}\right)=0, \quad p \in[1, \infty) .
$$

\section{REFERENCES}

[1] P. Billingsley, Convergence of Probability measures, John Wiley and Sons, New York-London-Sydney-Toronto, 1968.

[2] V.I. Bogachev, Gaussian Measures, Math. Surveys and Monographs, Vol. 62, Amer. Math. Soc., Providence, R.I., 1998.

[3] Z. Brzeźniak and J.M.A.M. van Neerven, Stochastic convolution in separable Banach spaces and the stochastic linear Cauchy problem, Studia Math. 143 (2000), 43-74.

[4] G. Da Prato, S. Kwapién, and J. Zabczyk, Regularity of solutions of linear stochastic equations in Hilbert spaces, Stochastics 23 (1987), 1-23.

[5] M.A. Krasnosel'skiı̌ and Ja.B. Rutickiı̌, CONVEX FUnCTIONS AND ORLICZ SPACES, Noordhoff Ltd., Groningen, 1961. 
[6] J.M.A.M. van Neerven, Continuity and representation of Gaussian Mehler semigroups, Potential Anal. 13 (2000), 199-211.

Department of Mathematics, The University of Hull, Hull Hu6 7RX, ENGLAND

E-mail address: Z.Brzezniak@maths.hull.ac.uk

School of Mathematics, The University of New South Wales, Sydney 2052, AUSTRALIA

E-mail address: B. Goldys@unsw.edu.au

Department of Applied Mathematical Analysis, Technical University OF DelfT, P.O. Box 5031, 2600 GA Delft, The Netherlands

E-mail address: J.vanNeerven@its.tudelft.nl 